ISSN 0103-8478

\title{
Influência do espaçamento e da população de plantas de sorgo sacarino em diferentes épocas semeadura
}

Influence of spacing and plant population for sweet sorghum in different seeding seasons

\author{
Pedro Gonçalves Fernandes ${ }^{I^{*}}$ André May ${ }^{I I}$ Fábio Cunha Coelho \\ Marina Chamon Abreu ${ }^{\mathrm{II}}$ Karina Mendes Bertolino ${ }^{\mathrm{II}}$
}

\section{RESUMO}

O trabalho objetivou avaliar a interferência do espaçamento entrelinhas e população de plantas sobre a produção do sorgo sacarino em duas épocas de semeadura, safra e safrinha. Os experimentos foram conduzidos na Embrapa Milho e Sorgo, em Sete Lagoas (MG), em Latossolo Vermelho Distrófico, na safra 2011/2012. Para as duas épocas de semeadura, as parcelas foram compostas por cinco espaçamentos entrelinhas (50; 60; 70; e $80 \mathrm{~cm}$ e linha dupla $100 \times 50 \times 50 \mathrm{~cm}$ ), e as subparcelas por quatro populações de plantas de sorgo sacarino (80.000, 100.000, 120.000 e 140.000 plantas ha ${ }^{-1}$ ). O delineamento utilizado foi em blocos ao acaso (DBC), com três repetições. Utilizou-se a variedade de sorgo sacarino BRS506. O experimento de safra foi implantado em novembro/2011 e de safrinha em março/2012. A época de semeadura no período de safra resultou em maior rendimento de matéria fresca de colmos, matéria fresca de biomassa total, massa de caldo e Brix do sorgo sacarino, independente do arranjo de plantas. O espaçamento de $50 \mathrm{~cm}$ entrelinhas resultou em maior produção de biomassa total e massa de caldo ha ${ }^{-1}$, independente da época de semeadura. O espaçamento duplo resultou em valores similares ao espaçamento de $80 \mathrm{~cm}$, para as principais variáveis produtivas, independente da época de semeadura. As populações de plantas não influenciaram o rendimento de matéria fresca de colmos e massa de caldo, independente da época de semeadura.

Palavras-chave: Sorghum bicolor, arranjo de plantas, manejo cultural, sistema de produção.

\section{ABSTRACT}

The research aimed to evaluate the effect of row spacing and plant population on the production of sweet sorghum in sowing season and off-season crop. The experiments were conducted at Embrapa Maize and Sorghum, Sete Lagoas (MG) in an Oxisol in the 2011/2012 season. For two seasons of sowing, the plots were composed of five row spacings $(50 ; 60 ; 70$ and $80 \mathrm{~cm}$ and double line $100 \times 50 \mathrm{~cm})$, and sub-plots for four plant populations of sweet sorghum (80.000, 100.000, 120.000 and 140.000 plants $\left.h a^{-1}\right)$. The experimental design was randomized blocks with three replications. It was used the variety of sweet sorghum BRS506. The experiment in the sowing season was implemented in November/2011 and the off-season in March/2012. The sowing season period resulted in higher fresh weight of total biomass, juice mass and Brix of sweet sorghum, regardless of plant arrangement. The row spacing of $50 \mathrm{~cm}$ resulted in higher total biomass and juice mass $h^{-1}$, regardless of the period sowing. The double spacing resulted in values similar to the row spacing of $80 \mathrm{~cm}$ for the main production variables, regardless of the period sowing. The plant population did not influence the yield of fresh mass of stems and juice mass, regardless of the period sowing.

Key words: Sorghum bicolor, plant arrangement, crop management, production systems.

\section{INTRODUÇÃO}

O setor sucroenergético brasileiro vem sofrendo com a alta crescente dos custos de produção e desdobra-se para encontrar alternativas com finalidade de reduzi-los, focando principalmente em aumento de rendimentos agrícola e industrial.

Nesse cenário, o sorgo sacarino apresentase como alternativa promissora para complementação no fornecimento de matéria-prima para indústria sucroenergética. O sorgo sacarino é o termo utilizado para descrever tipos de sorgo (Sorghum bicolor L. Moench) que apresentam altas concentrações de

\footnotetext{
ISetor de Grandes Culturas, Laboratório de Fitotecnia (LFIT), Centro de Ciências e Tecnologias Agropecuárias (CCTA), Universidade Estadual do Norte Fluminense Darcy Ribeiro (UENF), Avenida Alberto Lamego, 2000, Bairro Horto, 28013-620, Campos dos Goytacazes, RJ, Brasil. E-mail: pgfagro@gmail.com. *Autor para correspondência.

IEmbrapa Milho e Sorgo, Sete Lagoas, MG, Brasil.
} 
açúcar nos colmos. Cultivado em diversos países, têm larga adaptabilidade, tolerância à seca e podem produzir entre 40 a $70 \mathrm{t} \mathrm{ha}^{-1}$ de biomassa fresca, com o Brix variando de 16 até 23\% (ALMODARES \&HADI, 2009).

$\mathrm{O}$ alto teor de açúcares diretamente fermentáveis contido no colmo é comparado à canade-açúcar, com o benefício de obter esse rendimento em período mais curto, utilizando cultivares que variam o ciclo de 100 a 120 dias e propagado via sementes (MAY et al., 2012b).

O sorgo sacarino pode ser colhido durante a entressafra da cana-de-açúcar, beneficiando a indústria sucroalcooleira, que não ficaria sem matériaprima para a produção de etanol nesse período. Além disso, a elevada produção de biomassa e a antecipação da colheita em relação à cana-de-açúcar colocam o sorgo sacarino como excelente matériaprima para produção de etanol, tendo como primeira estratégia seu cultivo nas áreas de renovação, visando a antecipar o período de moagem em cerca de 45 dias (PARRELLA et al., 2010).

Estudos têm revelado que a redução do espaçamento entrelinhas para a cultura do sorgo sacarino resulta em ganhos de caracteres agronômicos, de importância para a produção de etanol. Para população de plantas, o resultado dos estudos nem sempre indica efeito sobre as principais variáveis de produção, em decorrência da variação ambiental e pelos distintos espaçamentos avaliados em cada estudo.

ALBUQUERQUE et al. (2010), ao avaliar os espaçamentos 50; 70; 90; e $110 \mathrm{~cm}$ e populações de $100.000,150.000,200.000$ e 250.000 plantas ha $^{-1}$ para as variedades de sorgo sacarino BRS 506 e BRS 507 em diferentes locais no Estado de Minas Gerais, observaram que o aumento da população em até 250.000 plantas ha ${ }^{-1}$ propiciou incrementos na produtividade de biomassa verde, porém, sem elevações na massa de colmo por hectare, devido à redução do diâmetro com o aumento do número de plantas por hectare.

Ao avaliar o cultivar de sorgo sacarino 'BR 506' em Pelotas - RS, EMYGDIO et al. (2011) obtiveram maior produção de colmos por hectare quando submetido ao espaçamento entrelinhas de $50 \mathrm{~cm}$, independentemente da população de plantas. A produtividade média de colmos foi de $70 \mathrm{tha}^{-1} \mathrm{em}$ espaçamento de $50 \mathrm{~cm}$ e $48 \mathrm{t} \mathrm{ha} \mathrm{a}^{-1}$ para o espaçamento de $70 \mathrm{~cm}$.

MAY et al. (2012a), avaliando diferentes arranjos de plantas para a cultivar de sorgo sacarino 'CMSXS 647', afirmaram que a redução no espaçamento de plantio apresenta maior influência no aumento da produção de biomassa fresca de colmos do que a densidade de plantas.

Assim, o presente trabalho teve por objetivo avaliar o efeito da combinação entre o espaçamento entrelinhas e a população de plantas do sorgo sacarino na região central do Estado de Minas Gerais, em condições de safra e safrinha.

\section{MATERIAL E MÉTODOS}

Os experimentos foram conduzidos na sede da Embrapa Milho e Sorgo, no município de Sete Lagoas, na região central do Estado de Minas Gerais, durante o período de 09 de novembro de 2011 a 16 de julho de 2012 .

O município de Sete Lagoas situa-se a

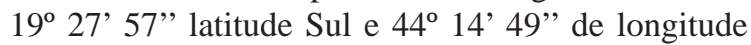
Oeste, com altitude média de 767 metros e relevo plano a levemente ondulado. Segundo a classificação de KÖPPEN (1948), o clima é classificado como Cwa, com inverno seco e verão úmido com chuva. A temperatura do ar média anual é de $21,1^{\circ} \mathrm{C}$ e a amplitude térmica está em torno de $6^{\circ} \mathrm{C}$. As temperaturas mais baixas são da ordem de $11,5^{\circ} \mathrm{C}$, registradas nos meses de junho e julho, as mais elevadas estão na faixa de 28,5 a $30^{\circ} \mathrm{C}$, verificadas nos meses de outubro a março. A estação seca se estende de maio a setembro e a estação chuvosa compreende o período dos meses de novembro a março, com precipitações mensais variando de 150 a 290mm aproximadamente. A precipitação pluvial total média anual histórica é de 1.384mm (GOMIDE et al., 2006).

O experimento foi conduzido em Latossolo Vermelho Distrófico, com drenagem moderada e textura argilosa. A tabela 1 apresenta a análise química do solo, anterior à instalação do experimento. A calagem não foi necessária, pois a saturação por bases encontrava-se no nível adequado para a cultura do sorgo sacarino, de acordo com ALVAREZ et al.(1999).

Foram instalados dois experimentos em parcelas subdivididas em duas épocas, a primeira época no período de safra e outra em safrinha. Em cada experimento, as parcelas foram compostas por cinco espaçamentos entrelinhas, sendo quatro simples, 50; 60; 70; e $80 \mathrm{~cm}$ e um em linha dupla $100 \times 50 \times 50 \mathrm{~cm}$, e as subparcelas por quatro populações de plantas de sorgo sacarino (80.000, 100.000, 120.000 e 140.000 plantas $\mathrm{ha}^{-1}$ ). O delineamento utilizado foi em blocos ao acaso (DBC), com três repetições. Cada unidade experimental foi constituída por quatro linhas de 
Tabela 1 - Aspectos químicos dos solos da área experimental, na profundidade de 0-20 cm, em Sete Lagoas - MG.

\begin{tabular}{|c|c|c|c|c|c|c|c|c|c|c|c|}
\hline $\mathrm{pH}$ & $\mathrm{H}+\mathrm{Al}$ & $\mathrm{Al}$ & $\mathrm{Ca}$ & $\mathrm{Mg}$ & SB & CTC & $\mathrm{K}$ & $\mathrm{P}$ & MO & $\mathrm{V}$ & $\mathrm{m}$ \\
\hline -------- & -------- & -1--- & molc & $-\cdots$ & 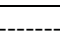 & -------- & \multicolumn{2}{|c|}{--------mg dm"------- } & $\mathrm{g} \mathrm{dm}^{-3}$ & -------- & ------- \\
\hline 6,4 & 5,38 & 0,02 & 4,3 & 0,8 & 5,3 & 10,7 & 107 & 32,9 & 2,7 & 50 & 0,34 \\
\hline
\end{tabular}

quatro metros de comprimento, espaçadas de acordo com o tratamento, onde foram consideradas como área útil, as duas linhas centrais, eliminando-se $50 \mathrm{~cm}$ de cada extremidade. Foi utilizada a variedade de sorgo sacarino BRS 506, desenvolvida pela Embrapa Milho e Sorgo.

O preparo do solo foi realizado convencionalmente, com uma aração e duas gradagens, antes da instalação dos experimentos. Os diferentes espaçamentos entrelinhas foram demarcados com auxílio de um trator acoplado a um sulcador "bico de pato”. Após a marcação, foi realizada adubação de base no fundo do sulco, na dose de $400 \mathrm{~kg} \mathrm{ha}^{-1}$ do formulado 8-28-16, equivalente a $32 \mathrm{~kg} \mathrm{ha}^{-1}$ de $\mathrm{N}, 112 \mathrm{~kg} \mathrm{ha}^{-1}$ de $\mathrm{P}_{2} \mathrm{O}_{5}$ e $64 \mathrm{~kg} \mathrm{ha}^{-1}$ de $\mathrm{K}_{2} \mathrm{O}$, baseado na perspectiva de produção de biomassa total superior a 60t ha-1 (ALVAREZ et al., 1999).

A semeadura foi realizada dia nove de novembro de 2011 para o experimento da safra e 15 de março de 2012 para o período de safrinha. As duas semeaduras foram realizadas manualmente com auxílio de réguas marcadoras. Em cada marcação, foram depositadas três sementes de sorgo sacarino, a fim de garantir a germinação de pelo menos uma plântula por marcação semeada e realizar o arranjo de plantas adequado de acordo com cada tratamento, realizando o raleio 15 dias após a semeadura (DAS), deixando-se apenas uma planta por cada ponto de semeio. A adubação de cobertura foi realizada para safra e safrinha quando as plantas estavam com 3-4 folhas definitivas, na dose de $400 \mathrm{~kg} \mathrm{ha}^{-1}$ de 20-02-20 aos 20 DAS e $90 \mathrm{~kg} \mathrm{ha}^{-1}$ de ureia aos 35DAS, para fornecimento total de $120 \mathrm{~kg} \mathrm{ha}^{-1}$ de $\mathrm{N}$ e $80 \mathrm{~kg} \mathrm{ha}^{-1}$ de $\mathrm{K}_{2} \mathrm{O}$. A cultura foi irrigada semanalmente por aspersão convencional, com lâmina de $10 \mathrm{~mm}$, de forma suplementar à precipitação local. O manejo fitossanitário foi realizado com produtos registrados para a cultura, conforme a necessidade de cada época de semeio.

A colheita dos experimentos de safra e de safrinha foi realizada 120DAS. As variáveis analisadas em cada parcela foram altura de plantas, medindo a distância média em metros da base rente ao solo até a extremidade das panículas, e diâmetro de colmo, medindo a distância média em milímetros no terço médio do colmo, ambas em dez plantas. Foi analisada a matéria fresca de colmos, pela pesagem em kg da massa fresca dos colmos da área útil, eliminando folha e panícula no momento que atingiram o ponto farináceo. Em seguida, realizouse a pesagem com folha e panícula para obter a matéria fresca de biomassa total, e ambas variáveis foram convertidas para $\mathrm{Mg}$ ha $^{-1}$. A variável massa de caldo ha-1 foi quantificada pela pesagem em $\mathrm{kg}$ do caldo extraído dos colmos de dez plantas colhidas na área útil, passadas uma única vez em moenda e, posteriormente, convertido em $\mathrm{Mg} \mathrm{ha}^{-1}$, e o ${ }^{\circ} \mathrm{Brix}$ foi determinado por refratômetro digital de leitura automática, em amostra do caldo extraído dos colmos de dez plantas.

Os dados foram submetidos, inicialmente, à análise de variância individual para os experimentos em safra e safrinha. Posteriormente, a partir das observações das grandezas do quadrado médio do resíduo de cada experimento, foi realizada a análise conjunta, pois o experimento de safra foi considerado homogêneo em relação ao de safrinha, de acordo com diferença menor que quatro vezes entre os quadrados médios do resíduo (BOX, 1954). Com o fator espaçamento, procedeu-se à comparação pelo teste Tukey para verificar o efeito do espaçamento duplo em relação aos simples e, posteriormente, realizou-se análise de regressão somente para os espaçamentos simples entrelinhas (RIBEIRO JÚNIOR, 2001). Para população de plantas, realizou-se análise de regressão. As análises estatísticas foram realizadas com o SAEG (Sistema para Análises Estatísticas e Genéticas), versão 8.0, desenvolvido pela FUNARBE, UFV, de Viçosa-MG.

\section{RESULTADOS E DISCUSSÃO}

A análise de variância conjunta dos experimentos demonstrou que houve efeito $(\mathrm{P}<0,05)$ da interação entre espaçamento e época para a variável altura de plantas. Assim procedeu-se o desdobramento para observar o efeito dos espaçamentos em cada época de plantio. Para o experimento de safra, não 
houve diferença significativa entre os espaçamentos avaliados, resultando em altura média de 2,93m. $\mathrm{Na}$ safrinha, houve diferença significativa entre os espaçamentos, mas o duplo não diferiu significativamente dos espaçamentos simples, resultando em plantas com 2,80m. Por outro lado, a altura de plantas decresceu linearmente com o aumento do espaçamento entrelinhas simples (Figura 1A). Assim, o aumento de $30 \mathrm{~cm}$ no espaçamento entrelinhas resultou em plantas $11,4 \mathrm{~cm}$ menores no plantio de safrinha.

Resultados semelhantes foram verificados por outros autores (MAY et al., 2012a; SNIDER et al., 2012), em experimentos de avaliação de arranjo de plantas utilizando outros genótipos de sorgo sacarino. Além disso, o resultado deste trabalho corrobora os resultados obtidos por esses autores na ausência de efeito da população de plantas sobre a altura de plantas.

As plantas cultivadas no espaçamento reduzido tiveram crescimento em altura maior, devido à menor competição entre as plantas na linha de cultivo, pela melhor distribuição espacial das plantas, favorecendo o aproveitamento dos fatores de produção disponíveis em cada época de semeio. MAY et al. (2012a) afirmaram que a competição por luz é uma das modalidades de interferência que provoca maior impacto sobre o crescimento nas plantas, pois restringe a fonte predominante de energia aos processos básicos de recrutamento de elementos e de elaboração de todas as substâncias envolvidas no crescimento do vegetal.

Para a variável diâmetro de colmos, houve efeitos da população de plantas $(\mathrm{P}<0,01)$ e espaçamento $(\mathrm{P}<0,05)$. Ocorreu relação linear negativa para o diâmetro de colmos, em função da população de plantas (Figura 1B). Com o aumento de 60.000 plantas ha-1 ${ }^{-1}$ observou-se decréscimo de 1,21mm no diâmetro de colmos, a partir de 80.000 plantas ha- ${ }^{-1}$. Esses resultados corroboram os obtidos por MAY et al. (2012b), os quais afirmaram que o aumento da população de plantas ha-1 pode resultar na redução do diâmetro de plantas. Os mesmos autores também afirmam que a redução de diâmetro do colmo se correlaciona positivamente com o acamamento e quebramento de plantas.

Para o fator espaçamento entrelinhas, o duplo diferiu significativamente dos espaçamentos de 50, 70 e $80 \mathrm{~cm}$, no entanto, não diferiu do espaçamento de $60 \mathrm{~cm}$, resultando em média 16,53mm de diâmetro de colmos. Para os espaçamentos simples entrelinhas, ocorreu relação quadrática para o diâmetro de colmos, em que o aumento do espaçamento até $63 \mathrm{~cm}$ entrelinhas, resultou em redução no diâmetro de colmos e, a partir deste ponto, ocorreu incremento desta variável (Figura 1C), proporcionado, possivelmente, pelo aumento na interceptação da radiação solar pelo dossel da planta, aumentando a disponibilidade de fotoassimilados (MAY et al., 2013).

Em relação à variável matéria fresca de colmos, houve efeito $(\mathrm{P}<0,01)$ para época de semeio, espaçamento entrelinhas e interação entre espaçamento e época de semeio. Observou-se relação linear, de modo que a matéria fresca de colmos reduziu com o aumento do espaçamento simples entrelinhas para as duas épocas de semeio (Figura 1D). Para o experimento de safra, a redução $80 \mathrm{~cm}$ para $50 \mathrm{~cm}$ no espaçamento entrelinhas resultou em aumento de 22,55Mg ha-1 da massa fresca de colmos e, no experimento conduzido em safrinha, o acréscimo foi de $10,71 \mathrm{Mg} \mathrm{ha}^{-1}$.

O espaçamento duplo na safra não diferiu $(\mathrm{P}<0,01)$ do espaçamento simples de $80 \mathrm{~cm}$, resultando em média 48,9 $\mathrm{Mgha}^{-1}$ de matéria fresca de colmos, sendo 33\% inferior ao espaçamento simples entrelinhas de $50 \mathrm{~cm}$. Na safrinha, o duplo não diferiu significativamente dos espaçamentos simples de 60, 70 e $80 \mathrm{~cm}$, resultando em média 43,10 $\mathrm{Mg} \mathrm{ha}^{-1}$, sendo $19 \%$ inferior ao espaçamento entrelinhas de $50 \mathrm{~cm}$. Esse incremento de matéria fresca com a redução do espaçamento entrelinhas também foi encontrado por ALBUQUERQUE et al. (2010), com as cultivares 'BRS506' e 'BRS507', e por MAY et al. (2012a) com a cultivar 'CMSXS647', em experimentos no Estado de Minas Gerais, além de SNIDER et al. (2012) com híbrido de sorgo forrageiro denominado 1990 da empresa Sorghum Partners ${ }^{\circledR}$, nos Estados do Alabama e Arkansas, nos Estados Unidos. Esse comportamento demonstra a alta influência que a alteração do total de metros lineares cultivados por hectare causa na produtividade de colmos por área cultivada e, consequentemente, a baixa capacidade de resposta que o sorgo sacarino apresenta para compensar o aumento do número de plantas na linha de cultivo.

Não houve influência do fator população de plantas na massa fresca de colmos para a cultivar estudada, corroborando os resultados obtidos por WORTMANN et al. (2010) e SNIDER et al. (2012), avaliando populações de 79.000 a 233.000 plantas por hectare sobre a produtividade de diferentes cultivares de sorgo.

MAY et al. (2012a), avaliando o efeito de diferentes arranjos de plantas da cultivar 'CMSXS647’ em Minas Gerais, observaram efeito da menor população de plantas avaliada, 80.000 plantas $\mathrm{ha}^{-1}$, associado ao menor espaçamento entrelinhas, 

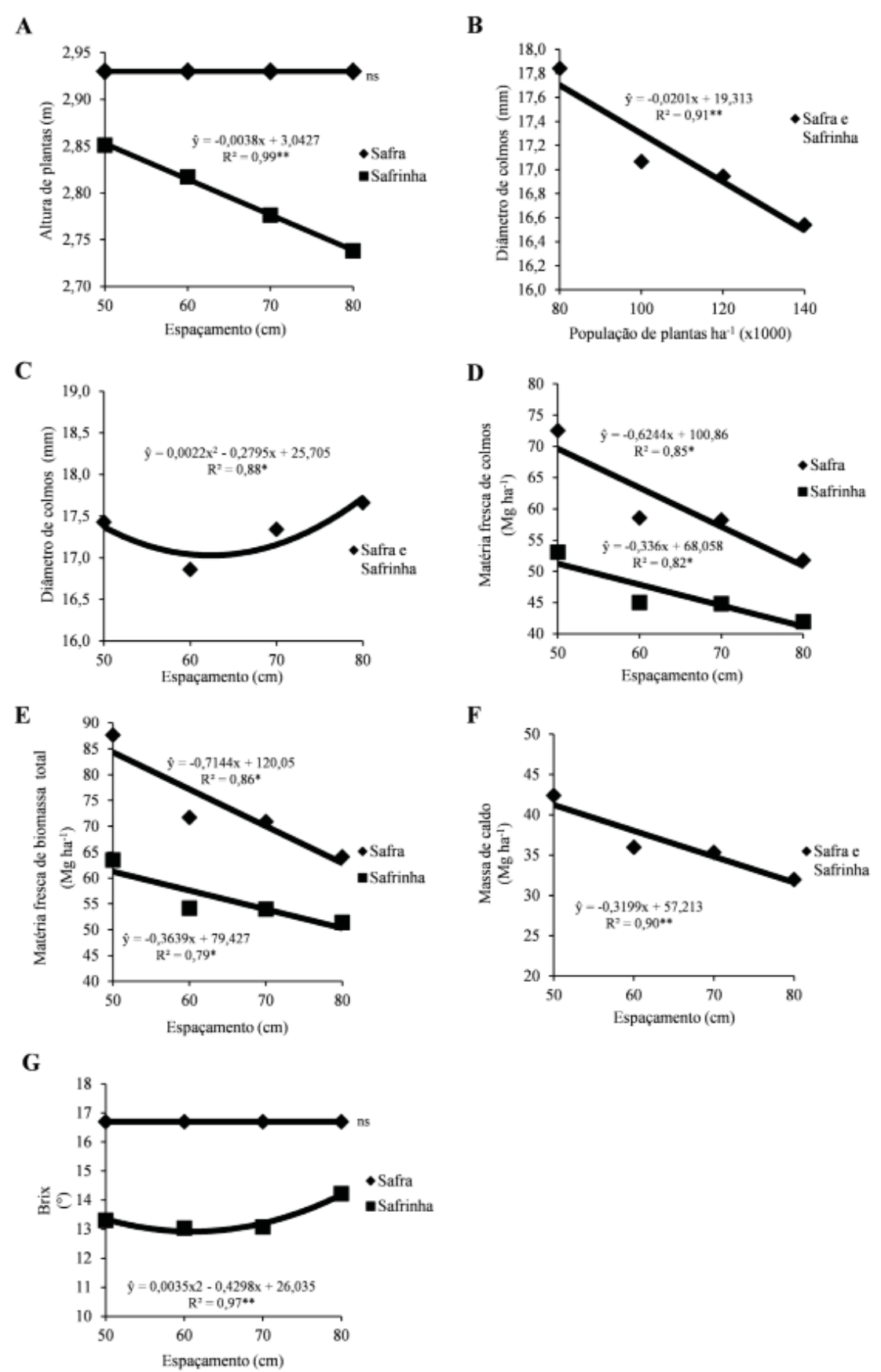

Figura 1 - Altura de plantas (A), diâmetro de colmos (C), matéria fresca de colmos (D), matéria fresca de biomassa total (E), massa de caldo (F) e Brix (G), considerando espaçamentos simples entrelinhas; diâmetro de colmos (B), considerando população de plantas em safra e safrinha da cultivar de sorgo sacarino 'BRS506', em Sete Lagoas - MG.

$50 \mathrm{~cm}$, resultando em menor rendimento. Assim, resultados de produtividade de colmos de sorgo sacarino por área cultivada em função da população de plantas podem diferir conforme ao ambiente e a cultivar estudada (WORTMANN et al., 2010).
O comportamento da variável matéria fresca de biomassa total foi similar ao observado para a matéria fresca de colmos, ocorrendo interação $(\mathrm{P}<0,01)$ entre os fatores espaçamento e época de semeio (Figura 1E). A massa de matéria fresca de 
biomassa total aumentou em 21,43Mg ha-1 com a redução de $80 \mathrm{~cm}$ para $50 \mathrm{~cm}$ do espaçamento simples entrelinhas no semeio de safra e $10,92 \mathrm{Mg}$ ha $^{-1}$ na safrinha. Na safra, o comportamento do espaçamento duplo não diferiu significativamente do espaçamento simples de $80 \mathrm{~cm}$, resultando em média $60,97 \mathrm{Mg} \mathrm{ha}{ }^{-1}$ de biomassa total, sendo $30 \%$ inferior ao espaçamento simples de $50 \mathrm{~cm}$. No experimento conduzido em safrinha, o espaçamento duplo não diferiu significativamente dos espaçamentos simples de 60,70 e $80 \mathrm{~cm}$, resultando, em média, 52,82 $\mathrm{Mg} \mathrm{ha}^{-1}$, sendo $17 \%$ inferior ao espaçamento simples de $50 \mathrm{~cm}$.

A variável massa de caldo sofreu efeito altamente significativo do espaçamento entrelinhas $(\mathrm{P}<0,01)$. A massa de caldo diminuiu linearmente com o aumento de $50 \mathrm{~cm}$ para $80 \mathrm{~cm}$ entrelinhas, resultando em decréscimo de $9,60 \mathrm{Mg} \mathrm{ha}^{-1}$ (Figura1F). $\mathrm{O}$ espaçamento duplo não diferiu significativamente do espaçamento simples de $80 \mathrm{~cm}$, resultando em média $30,54 \mathrm{Mg}$ ha $^{-1}$ de massa, sendo $28 \%$ menor em relação ao espaçamento de $50 \mathrm{~cm}$, que acumulou maior massa de caldo.

O Brix foi afetado pela época de semeio, espaçamento entrelinhas simples e interação entre esses fatores $(\mathrm{P}<0,01)$. Para o experimento conduzido na safra, não houve diferença significativa entre os espaçamentos avaliados (Figura 1G). Na época de semeio em safrinha, houve relação quadrática, pois o aumento do espaçamento entrelinhas de $50 \mathrm{~cm}$ até $60 \mathrm{~cm}$, reduziu o Brix e, a partir desse espaçamento, ocorreu acúmulo de sólidos solúveis totais. O espaçamento duplo resultou em $2,57^{\circ}$ Brix a mais, em relação à média dos espaçamentos simples de 50, 60 e $70 \mathrm{~cm}$ entrelinhas, que resultaram em menor acúmulo. Vale ressaltar que, independente do espaçamento entrelinhas, o experimento semeado na safra resultou em caldo com 20,17\% mais sólidos solúveis totais, em relação ao conduzido em safrinha.

Esse comportamento possivelmente ocorreu devido às melhores condições climáticas, como temperaturas médias mais elevadas, não atrasando o desenvolvimento da planta, com consequente menor susceptibilidade à incidência de pragas e doenças foliares (MOURÃO et al., 2012; SILVA, et al., 2012), resultando em plantas bem desenvolvidas, com maiores ganhos em variáveis quantitativas e qualitativas.

\section{CONCLUSÃO}

A época de semeadura no período de safra resultou em maior rendimento de matéria fresca de colmos, matéria fresca de biomassa total, massa de caldo e o Brix do sorgo sacarino, independente do arranjo de plantas. O espaçamento de $50 \mathrm{~cm}$ entrelinhas resultou em maior produção de biomassa total e massa de caldo ha-1 ${ }^{-1}$ independente da época de semeio. O espaçamento duplo resultou em valores similares ao espaçamento de $80 \mathrm{~cm}$ para as principais variáveis produtivas, independente da época de semeio. As populações de plantas não influenciaram o rendimento de matéria fresca de colmos e massa de caldo, independente da época de semeio.

\section{REFERÊNCIAS}

ALBUQUERQUE, C.J.B. et al. Potencial forrageiro de cultivares de sorgo sacarino em diferentes arranjos de plantas e localidades de Minas Gerais. In: CONGRESSO NACIONAL DE MILHO E SORGO, 28.; SIMPÓSIO BRASILEIRO SOBRE LAGARTA DO CARTUCHO, 4., 2010, Goiânia, GO. Potencialidades, desafios e sustentabilidade: resumos expandidos. Goiânia: ABMS, 2010. 1 CD-ROM.

ALMODARES, A.; HADI, M.R. Production of bioethanol from sweet sorghum: a review. African Journal of Agricultural Research, v.4, n.9, p.772-780, 2009.

ALVAREZ, V.H.et al. Interpretação dos resultados das análises de solos. In: RIBEIRO, A.C. et al. (Ed.). Recomendações para o uso de corretivos e fertilizantes em Minas Gerais - 5 a Aproximação. Viçosa: UFV, 1999. p.25-32.

BOX, G.E.P. Some theorems on quadratic forms apllied in the study of analysis of variance problems, I. Annals of Mathematical Statistics. v.25, p.290-302, 1954. Disponível em: <http://dx.doi. org/10.1214/aoms/1177728717>. Acesso em: 26 jan. 2013. doi: 10.1214/aoms/1177728717.

EMYGDIO, B.M. et al. Desempenho de cultivares de sorgo sacarino para a produção de etanol sob diferentes densidades de plantas. Pelotas, RS: Embrapa Clima Temperado, 2011. 22p. (Boletim de pesquisa e desenvolvimento)

GOMIDE, R.L.et al. Caracterização climática e determinação da necessidade hídrica de culturas do sítio-específico de precisão de Sete Lagoas para a fenotipagem de genótipos de cereais tolerantes à seca. Sete Lagoas, MG, 2006. 7p. Acesso em:4 dez. 2012. Online. Disponível em: <http://www.alice.cnptia. embrapa.br/handle/doc /490096>.

KÖPPEN, W. Climatologia: conunestudio de los climas de latierra. Mexico:Fondo de Cultura Economica, 1948. 478p.

MAY, A. et al. Influência do arranjo de plantas no desempenho produtivo de sorgo sacarino (Sorghum bicolor (L.) Moench), em Sete Lagoas-MG. In: CONGRESSO NACIONAL DE MILHO E SORGO, 29. 2012. Águas de Lindóia, SP, Anais... Águas de Lindóia:ABMS, 2012a. p.2382-2389. 1 CD-ROM.

MAY, A.et al. Manejo e tratos culturais. In: et al. (Ed.). Sistema Embrapa de produção agroindustrial de sorgo sacarino para bioetanol: Sistema BRS1G-Tecnologia Qualidade Embrapa. Sete Lagoas: Embrapa Milho e Sorgo, 2012b. p.22-31.

MAY, A. et al. Cultivo de sorgo sacarino em áreas de reforma de canaviais. Sete Lagoas: Embrapa Milho e Sorgo, 2013.36p. (Circular Técnica, 186). 
MOURÃO, C.S.et al. Efeito de doenças foliares no desenvolvimento de sorgo sacarino. In: CONGRESSO NACIONAL DE MILHO E SORGO, 29. 2012. Águas de Lindóia, SP, Anais... Águas de Lindóia:ABMS, 2012a. p.749-755. 1 CD-ROM.

PARRELLA, R.A.C. et al. Desempenho de cultivares de sorgo sacarino em diversos ambientes visando produção de etanol. In: CONGRESSO NACIONAL DE MILHO E SORGO, 28., 2010, Goiania, Go. Anais... Goiânia:ABMS, 2010. p.256-263.

RIBEIRO JÚNIOR, J.I. Análises Estatísticas no SAEG. José Ivo Ribeiro Júnior. Viçosa: UFV, 2001.301p.

SILVA, D.D.et al. Controle de doenças. In: MAY, A. et al. (Ed.). Sistema Embrapa de produção agroindustrial de sorgo sacarino para bioetanol: Sistema BRS1G-Tecnologia Qualidade Embrapa. Sete Lagoas: Embrapa Milho e Sorgo, 2012. p.68-84.

SNIDER, J.L. et al. The effect of row spacing and seeding rateon biomass production and plant stand characteristics of non-irrigated photoperiod-sensitive sorghum (Sorghum bicolor (L.) Moench). Industrial Crops and Products, v.37, p.527-535, 2012. Disponível em: <http://dx.doi.org/10.1016/j.indcrop.2011.07.032>. Acesso em: 20 jan. 2013. doi: 10.1016/j.indcrop.2011.07.032.

WORTMANN, C.S. et al. Dryland performance of sweet sorghum and grain crops for biofuel in Nebraska. Agronomy Journal, v.102, p.319-326, 2010. Disponível em: <http://dx.doi. org/10.2134/agronj2009.0271>. Acesso em: 28 jan. 2013. doi: 10.2134/agronj2009.0271 